\title{
The Performance of a Novel Latching-Type Electromagnetic Actuator for Single-Port Laparoscopic Surgery
}

\author{
Haochen Wang, Ali El Wahed* \\ Mechanical Engineering, University of Dundee, Dundee, UK \\ Email: *a.elwahed@dundee.ac.uk
}

How to cite this paper: Wang, $\mathrm{H}$. and $\mathrm{El}$ Wahed, A. (2019) The Performance of a Novel Latching-Type Electromagnetic Actuator for Single-Port Laparoscopic Surgery. Journal of Modern Physics, 10, 1659-1673.

https://doi.org/10.4236/jmp.2019.1014109

Received: October 25, 2019

Accepted: December 1, 2019

Published: December 4, 2019

Copyright $\odot 2019$ by author(s) and Scientific Research Publishing Inc. This work is licensed under the Creative Commons Attribution International License (CC BY 4.0).

http://creativecommons.org/licenses/by/4.0/

\section{(c) () Open Access}

\begin{abstract}
This paper reports on the performance evaluation of a novel latching-type electromagnetic actuator which is designed to be embedded at selected joints along single-port laparoscopic surgical instruments (SLS). The aim of this actuator is to allow these instruments to become articulated with a push of a button in order to provide the optimum angulation required during SLS operations. This new actuator is comprised of electromagnetic coil elements, soft magnetic frames and a permanent magnet. Unlike conventional electromagnetic actuators, latching-type electromagnetic actuators could maintain their positions at either end of the actuation stroke without any power application requirement. In the current design, magnetic attraction forces initiated between the permanent magnet and the magnetic frame are utilised to lock the position of the actuator whilst a certain angulation position of the actuator is achieved as a result of the magnetic repulsion forces established between the permanent magnet and the coil elements. The performance of the new actuator in terms of the output force, maximum angulation and patient's safety, was evaluated experimentally and the results were found to compare well with those acquired numerically using finite element methods. This actuator was seen to exhibit sufficient actuation forces and hence, it was capable of providing adaptable angulation characteristics for SLS tools. Finally, thermal evaluation of the actuator's operation was conducted, which was found to be within safety limits specified by clinicians.
\end{abstract}

\section{Keywords}

Latching Electromagnetic Actuator, Laparoscopic Surgical Tools Angulation

\section{Introduction}

Single-port laparoscopic surgery (SLS), which utilises one major incision instead 
of multiple incisions, has become increasingly popular in the health service in recent years. Compared with the conventional multi-port laparoscopic surgery, SLS has many advantages such as less postoperative pain, early return of gastrointestinal function, shorter length of hospital stay and fewer blood losses [1]. However, SLS suffers from several problems such as inadequate exposure of the surgical field, surgical instrument crowding and lack of internal maneuverability for surgical tools, which have impeded the advancement of the SLS technique [1] [2] [3]. In addition, concerns have been raised that these problems have increased the length of operative times, which may potentially increase the risk of SLS operations for patients [4]. In order to solve some of the above SLS problems, several attempts have been carried out, for example, to improve the flexibility of SLS surgical instruments using a robotic platform [5], to direct the SLS camera using external magnetic fields [6] and to use pre-bent surgical instruments [7]. However, the sophisticated robotic instruments add to the cost and complexity of the surgery, and the time of training [8] [9] [10]. In addition, most of the developed tools could not be used as universal tools since they have their own limitations, including insufficient angulation and deficient force or torque output [11]. Consequently, a more practical approach is now necessary to advance SLS procedures. In this activity, the authors have been engaged in the development of novel latching-type electromagnetic actuators that could be embedded along SLS tools with the aim to enhance their levels of stiffness and degrees-of-freedom.

The applications of electromagnetic actuators in medical devices have seen a surge in recent years [12] [13] [14] [15]. This is attributed mainly to their relatively low magnetic energy that is deemed safe for patients as well as their uncomplicated fabrication and after use sterilisation processes. However, in order to introduce the electromagnetic actuator technology into the optimisation of SLS instruments, some difficulties need to be addressed including the remarkable reduction of the force output with the miniaturisation of these actuators [15] [16]. In addition, the inevitable heat buildup caused by the continuous current feed to energise the magnetic circuit of these actuators has been found in certain medical applications as a problem since it breaches the patient's safety [17]. This problem was overcome in some applications using latching-type electromagnetic actuators, which utilise a permanent magnet to lock the position of the actuator instead of the magnetic energy generated by the continuous activation of the actuator's electromagnetic circuit [18]. However, these new generations of electromagnetic actuators, which incorporate springs to aid their actuation, were aimed for relatively large applications, such as the actuation of valves of internal combustion engines [18]. Therefore, in order to miniaturise the existing latching-type electromagnetic actuators and make them suitable for SLS applications, a comprehensive work is required to optimise their structure, material and excitation level.

This paper reports on the performance evaluation of a new miniaturised latching-type electromagnetic actuator which utilises a permanent magnet and 
dual-coil design for position locking and actuation, respectively. This new actuator is proposed to be embedded along SLS surgical instruments, which can be squeezed through a standard tocar-port, to enable the tool's angulation that is required during SLS operations. Special SLS requirements such as the trocar-port size, central channel for passing a cable to control the end effector, force output level and angulation degree rather than just a linear stroke have all been considered in the design of the new actuator. In this study, the performance of the new actuator under various input conditions was assessed using numerical and experimental approaches. A thermal camera was also employed to evaluate the optimum duty cycle of the actuator operation, which ensures patients safety. Finally, the overall performance of the new actuator is discussed and compared with other competitive actuators.

\section{Actuator Design and 0ptimisation}

The new latching-type electromagnetic actuator, Figure 1, comprises of two electromagnetic coil elements, one at either end of its central chamber, a permanent magnet armature which could slide along the central chamber and a solid shell made from a soft magnetic material. This actuator was designed to be cylindrical in shape in order to match the shape of SLS trocar port. Also, a central channel was allowed in the design of the new actuator which spans its whole length to accommodate a guide wire that is used to control the end effector of the surgical instrument. In addition, an air gap feature was allowed in the magnetic circuit of the actuator with the aim to enhance the magnetic interaction between the coil elements and the permanent magnet. Furthermore, a solid titanium sliding element was added on the side of the actuator shell, which is linked to the side of the permanent magnet armature to transmit its linear actuation into a bending action using a swivelling element at the top of the actuator. Since the new actuator is proposed to be embedded along SLS tools, the above arrangement should enable the controlled angulation of these tools as necessary during SLS operations.

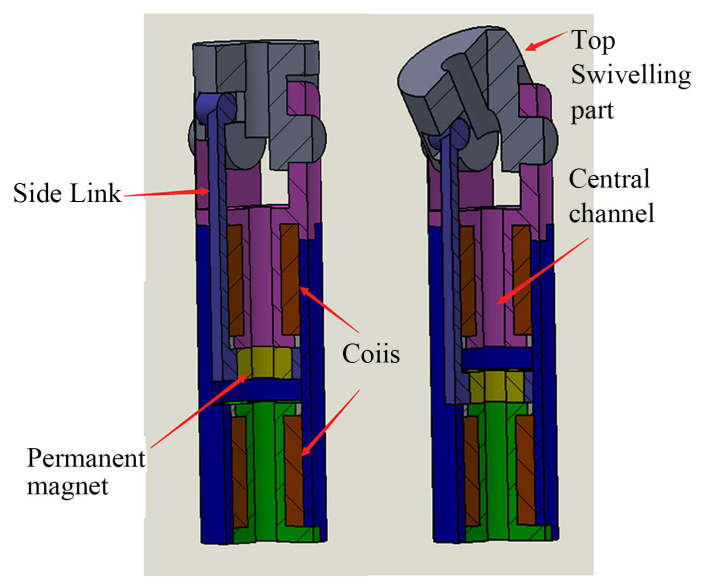

Figure 1. Cross-section views of the new actuator in its straight (left) and bending (right) positions. 
The new actuator utilises the magnetic force established between the permanent magnet and the soft magnetic ends of the central chamber to maintain the position of the armature during the deactivated status of the actuator. When an actuation is required, the coil that is close to the permanent magnet is supplied with a short current pulse to generate an opposite magnetic field which is designed to neutralise and overcome the magnetic field of the permanent magnet. As a result, the permanent magnet is subjected to a repelling magnetic force which drives it towards the other end of the central chamber. Once the armature completes its full stroke and is in contact with the other chamber end, it again becomes firmly locked in its new position under the effect of the magnetic attraction forces with the chamber end while the coil is deactivated. Consequently, the transformation of the armature between the two locking positions should generate the required angulation of the actuator end, which is used in the current application to provide SLS tools with the necessary bending output.

In order to optimise the design of the new actuator, 3-D electromagnetic finite element analyses were carried out using Ansys Workbench software (Version 17.0) with its associated magnetostatic module. The actuator 3-D model was created using Solidworks CAD software (Version 2016) and subsequently exported into the Ansys Workbench environment. The components of the 3-D model were set up with their specific properties and then meshed with a maximum element length of $0.0002 \mathrm{~m}$ as shown in Figure 2. The permanent magnet armature was assumed to travel statically along its imaginary stroke between the two ends of the central chamber, whilst the total magnetic force acting on the permanent magnet and the corresponding coil-energising current were recorded as a function of the stroke path length.

The most important design factors that are expected to critically affect the overall performance of the developed actuator were identified, which included the size of the electromagnetic coils and their excitation level in addition to the size of actuator's outer shell, height of the actuation channel and the size and grade of the permanent magnet.

A step-by-step optimisation process of the above parameters was carried out, which is summarised in Figure 3. It was found that the size of the electromagnetic components and the excitation level of the coil elements are proportional to the output of the actuator before the magnetic saturation limit of the actuator material was reached. The systematic actuator optimisation process eventually made it possible for a balance between the size and the output of the actuator to be achieved. The main parameters of the optimised actuator are summarised in Table 1.

Using a conventional CNC machining facility, the new actuator was then manufactured. The machined magnetic parts were subsequently annealed in order to regain the original magnetic properties of the material and also to remove any carbon residue from the surface of the parts. Figure 4 shows the manufactured actuator. 
A conservative model was developed to estimate the effects of the short current pulse on the temperature rise of the actuator, and the operating duty cycle. The heat generated by the coil, $W$ subjected to a direct current pulse feed, which is equivalent to the power consumption of the coil element during one complete period of the duty cycle, was estimated using:

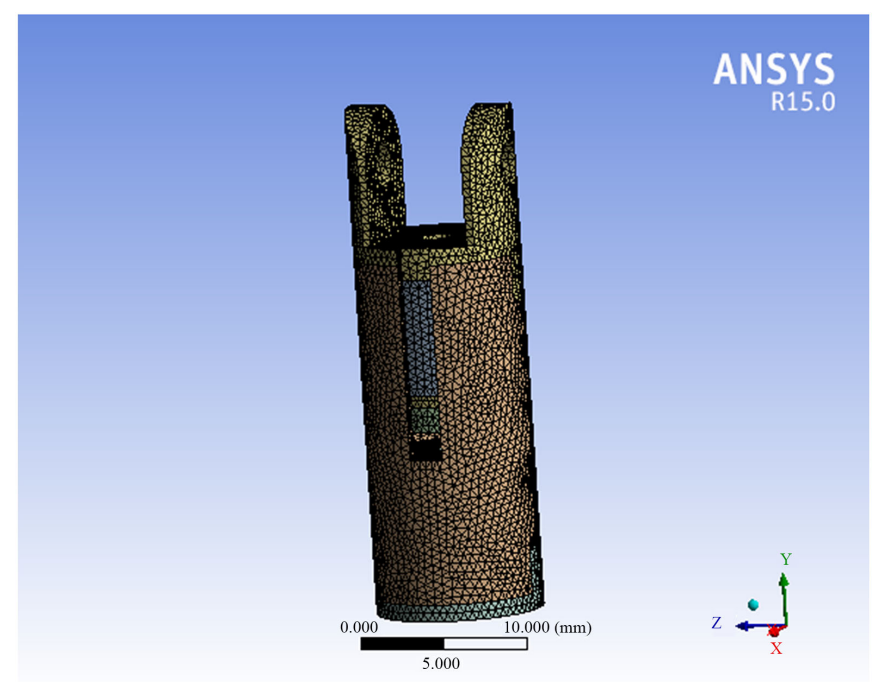

Figure 2. Mesh of the actuator model.

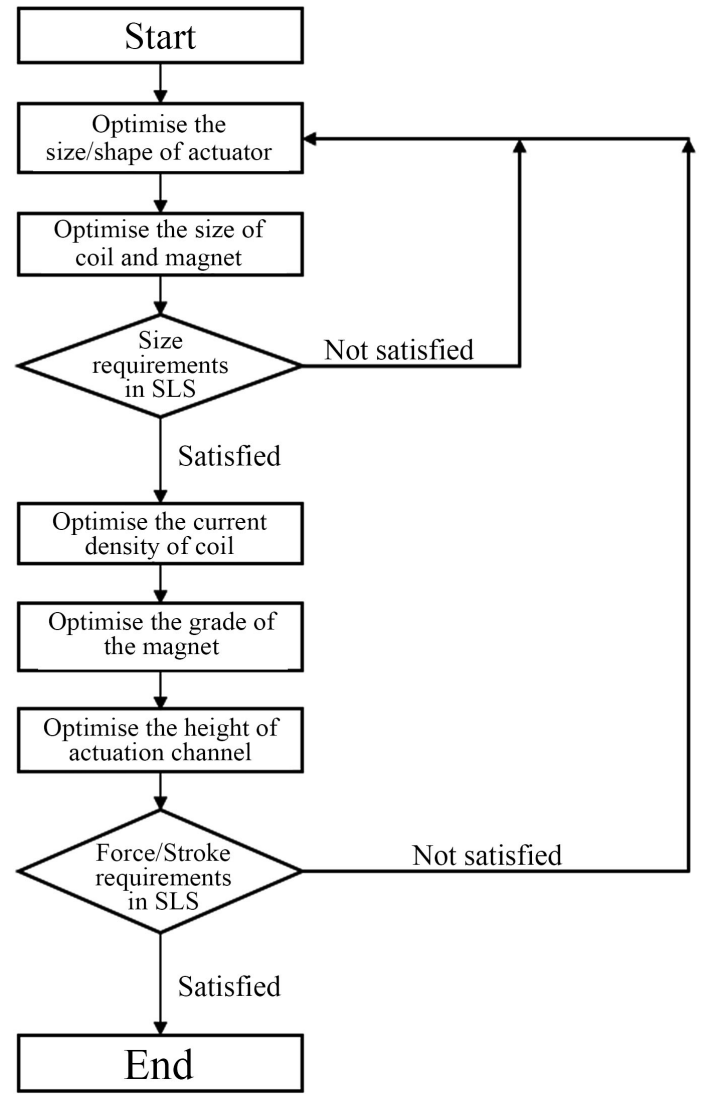

Figure 3. Flowchart of the new actuator design optimisation procedure. 


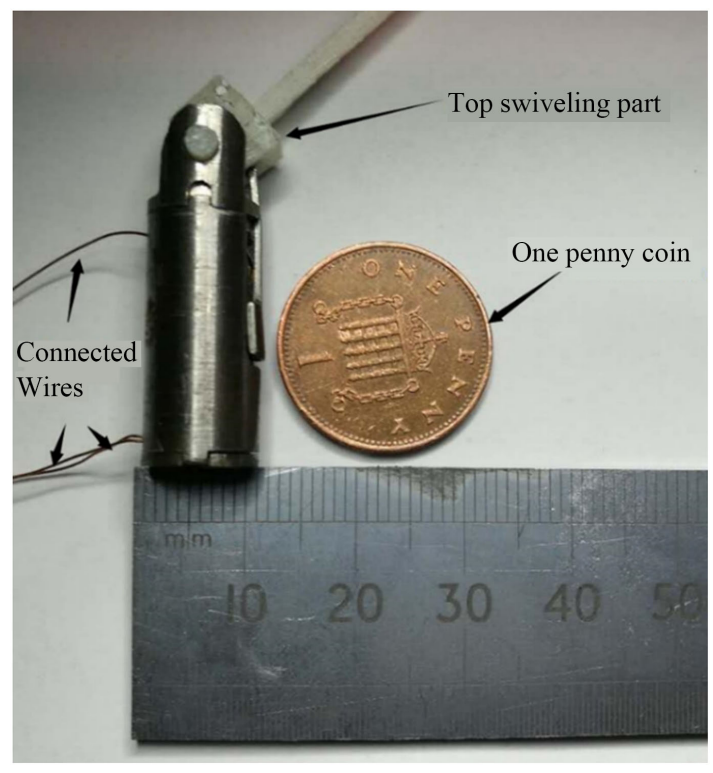

Figure 4. Prototype of new latching-type electromagnetic actuator shown next to a penny coin.

Table 1. Parameters of the optimised actuator.

\begin{tabular}{|c|c|}
\hline \multicolumn{2}{|c|}{ COIL } \\
\hline Coil Wire Diameter & $0.29 \mathrm{~mm}$ \\
\hline Number of Wire Turns per Coil & 192 \\
\hline Applied Current & $6.7 \mathrm{~A}$ \\
\hline Coil Current Density & $80,000 \mathrm{kA} / \mathrm{m}^{2}$ \\
\hline Conducting Area & $17.5 \mathrm{~mm}^{2}$ \\
\hline \multicolumn{2}{|c|}{ PERMANENT MAGNET } \\
\hline Inner Diameter & $2 \mathrm{~mm}$ \\
\hline Outer Diameter & $8 \mathrm{~mm}$ \\
\hline Thickness & $2 \mathrm{~mm}$ \\
\hline Grade & Neodymium N52 \\
\hline \multicolumn{2}{|c|}{ ACTUATOR } \\
\hline Outer Diameter & $10 \mathrm{~mm}$ \\
\hline Height & $31.6 \mathrm{~mm}$ \\
\hline Central Channel Diameter & $2 \mathrm{~mm}$ \\
\hline Maximum Linear Stroke of Magnet & $1.5 \mathrm{~mm}$ \\
\hline Shell Material & MaxiMag Low-Carbon Magnetic Iron \\
\hline
\end{tabular}

$$
W=I_{\max }^{2} R_{1} t_{1}
$$

where $t_{1}$ is the current pulse width that is also the duration during which the circuit is open, $I_{\max }$ is the peak value of the direct short current pulse and $R_{1}$ is the maximum resistance of the electromagnetic coil at the peak temperature. 
The temperature rise, $\Delta T$ of the coil element is given by [19]:

$$
\Delta T=\frac{W}{\lambda S t_{2}}=\frac{I_{\max }^{2} R_{1} t_{1}}{\lambda S t_{2}}
$$

where $\lambda$ is the heat dissipation coefficient, $S$ is the surface area of the coil and $t_{2}$ is the heat dissipation (temperature decay) time which is the sum time of one duty cycle. The duty cycle, $D$ is then approximated by the ratio $t_{1} / t_{2}$ [19], which is also given by:

$$
D=\frac{t_{1}}{t_{2}}=\frac{\Delta T \lambda S}{I_{\max }^{2} R_{1}}
$$

The relationship between the heat dissipation coefficient, $\lambda$ and the temperature rise, $\Delta T$ could then be expressed by [19]:

$$
\lambda=0.039 \Delta T+8.83
$$

By substituting Equation (3) into Equation (2), the optimum operating duty cycle under various operational conditions could then be estimated. The determination of the optimum operating duty cycle is essential in the current medical application since any operation that is more frequent than this duty cycle may result in an elevated coil temperature which could cause potential problems for the patients.

\section{Experimental Arrangement}

Various experimental protocols were designed to allow the systematic assessment of the performance of the new actuator, which included the least activation current tests, magnetic force tests and thermal tests aimed for the evaluation of the optimum working duty cycle of the actuator. The overall performance of the new actuator was then identified by analysing the results of the above tests under different conditions. A special circuit was designed to control the width of the short current pulse (between $0.1 \mathrm{~s}$ and $1 \mathrm{~s}$ ), which is required for the excitation of the electromagnetic coils of the actuator. After several trials, it was found that the actuation performance of the actuator was not significantly affected when the current pulse width was varied along the above range, which indicates that the response time of the actuator is faster than $0.1 \mathrm{~s}$ as a result of the small linear stroke of the actuator. Hence, and aiming for a reduction in the temperature of the electromagnetic circuit of the actuator, the current pulse width, $t_{1}$ was fixed at $0.1 \mathrm{~s}$ in all subsequent experiments.

\subsection{Least Activation Current Test Setup}

In this study, the least activation current of the electromagnetic coil of the new actuator is defined as the minimum applied current that is required to generate a magnetic field capable of releasing the permanent magnet from a locking status, which should then be ready to move along the sliding central chamber with a further current supply. Under this current level, there should be a balance between the magnetic forces generated by the permanent magnet and the electro- 
magnetic coil. In this test, ReaseJoy (Model QW-MS3010D) adjustable power source in addition to the current pulse width controlling circuit were used to energise the electromagnetic coil of the actuator whilst an Agilent Technologies (Model U3401A) digital multi-meter was used to record the peak value of the applied current. In this arrangement, the flat end of the shell of the actuator was connected to a solid horizontal platform while the permanent magnet armature was allowed to be attracted and attached to the upper end of the central chamber. Also, the permanent magnet was disconnected from the solid sliding link of the actuator in order to reduce any friction between the permanent magnet and the actuator shell. The applied pulse current was then gradually increased in steps of $0.1 \mathrm{~A}$ until the permanent magnet becomes detached from the central chamber upper end, which then drops towards the other end of the chamber with further current increase. Therefore, the peak current, which was recorded when the permanent magnet armature becomes detached from the upper end of the central chamber, was considered as the least activation current of the electromagnetic coil element.

In order to confirm the validity of this test, the procedure was repeated several times when the gap between the permanent magnet and the upper end of the central chamber was gradually increased using PTFE layers with specific thicknesses. The measured least activation current results were also compared with the numerical current results that were obtained from finite element simulations which will be detailed in Section 4.1 .

\subsection{Magnetic Force Test Setup}

Magnetic force tests were conducted using a Tinius Olsen tensile machine, model H5KS. The shell of the actuator was vertically aligned and fixed to the upper $10 \mathrm{~N}$ load cell of the machine whilst a non-magnetic metallic rod, which was supported by the platform of the machine, was routed through the central channel of the actuator and bonded to the permanent magnet. As a result, the position of the permanent magnet along the central sliding chamber could be controlled through precision axial input displacements that were supplied by the tensile machine and the corresponding total magnetic force acting on the permanent magnet was measured by the load cell of the machine. Figure 5 shows this experimental arrangement.

In this test, the ReaseJoy power supply as well as the current pulse width controlling circuit were used to energise the actuator, and the total magnetic force on the permanent magnet was measured for various positions along the actuation stroke for the two cases when the coil was activated and deactivated. The results of this test were compared with the numerical results that were obtained from the same finite element method which will be detailed in Section 4.1.

\subsection{Thermal Test Setup}

A Micro-Epsilon infrared thermal camera, model thermoIMAGER TIM 160 was 


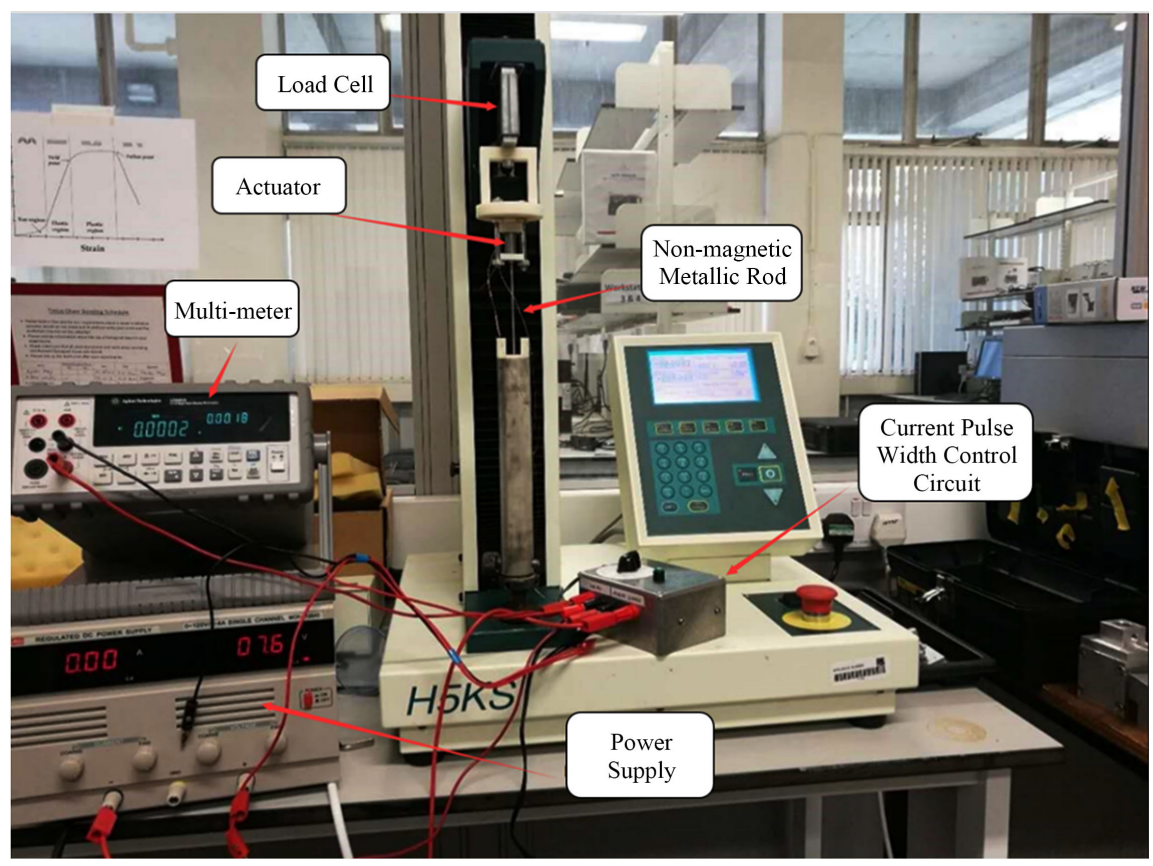

Figure 5. Magnetic force test setup.

employed to evaluate the thermal performance of the new actuator. The camera was placed in front of the actuator which was energised using the same circuit that was detailed in Section 3.1. In order to analyse the effect of the current excitation on the thermal load of the actuator, the coil element was supplied with a continuous current of $6.7 \mathrm{~A}$ until the thermal camera recorded a maximum temperature of $50^{\circ} \mathrm{C}$ in the detection area (nearby the upper coil position) of the actuator after which the current supply was stopped and the actuator was allowed to cool down naturally while the camera was still recording. The ambient temperature during these experimental investigations was maintained at $27^{\circ} \mathrm{C}$. Thermal analysis has also been conducted using Ansys Workbench software which will be detailed in Section 4.3. The thermal test results were used to evaluate a proper working duty cycle of the actuator that maintains the safety of patients during the operation of this actuator in SLS environments within tolerable levels.

\section{Results and Discussion}

In order to maintain consistency in this investigation, it was decided to allow the permanent magnet to start its stroke from the top-end of the central chamber in all the tests reported in this section.

An ISO-Tech oscillator, model IDS 8062, was employed to measure the rise time of the pulse current excitation of the actuator's electromagnetic coil. It was found that the average rise time is under $2 \mu$ s for pulse current excitations up to 6.7 A, which is negligible compared with the pulse current width that is around $100,000 \mu \mathrm{s}(0.1 \mathrm{~s})$. Therefore, the effect of the rise time of the applied pulse current on the results was not taken further into account in the subsequent tests. 


\subsection{Least Activation Current}

The least activation current was measured for various gap thicknesses between the permanent magnet and the upper end of the central chamber, which were created by the addition of PTFE layers and the results are shown in Figure 6 . The thickness of this gap was increased gradually up to a total thickness of 0.75 $\mathrm{mm}$ which represents half of the complete linear actuation stroke.

Also, a finite element analysis (FEA) using Ansys software was carried out to estimate comparable least activation current values which are also presented in Figure 6. This FEA model utilised the Ansys "Edge-Based" analysis method and a 3-D 20-node element, type "Solid 236", which is capable of modelling electromagnetic problems. In addition, a boundary, which was represented by a spherical air ball of $0.04 \mathrm{~m}$ diameter and subjected to a magnetic flux parallel condition of $0 \mathrm{mV}$, was allowed to encircle the actuator model. Moreover, material properties such as the B-H curve of the MaxiMag steel [20] as well as the coercive force and the residual induction of the permanent magnet [21] were subsequently specified in the FEA environment. Finally, the upper coil element was chosen as the source conductor in these FEA analyses. The least activation current values were then estimated using the Ansys FEA model following the same procedure that was applied to measure them experimentally. In particular, the supplied current to the upper coil element was gradually increased until the total force estimated on the permanent magnet armature was approximately zero \pm $0.01 \mathrm{~N}$. This current was then recorded as the least activation current which should enable the permanent magnet to detach itself from the upper end of the central chamber and subsequently slides towards the other end of the chamber with further current increase.

It can be seen, Figure 6 that for a zero gap, a current of about $3.3 \mathrm{~A}$ was required to energise the upper end coil element of the actuator in order to generate the required magnetic field that was sufficient to neutralise the permanent magnet field effect which initially permitted the locking status. However, when a gap of about $0.75 \mathrm{~mm}$ thick was allowed between the permanent magnet and the upper end of the central chamber, zero magnetic force was estimated on the permanent magnet without any current application as the magnet was exactly in the middle between the two ends of the central chamber and hence, the attraction forces established between the permanent magnet and the two ends balanced each other. Furthermore, it can be noted from Figure 6 that the numerical and experimental results are in a good agreement, indicating the validity of the employed Ansys finite element modelling technique.

\subsection{Actuator Magnetic Force}

Systematic testing of the new actuator was carried out and it was apparent that the optimum operation of the actuator was achieved when the current density of the electromagnetic coil element was approximately $80,000 \mathrm{kA} / \mathrm{m}^{2}$, which corresponds to an applied current of about 6.7 $\mathrm{A}$. The total magnetic force acting on 
the permanent magnet armature was recorded along the linear actuation stroke for the two cases when the coil was deactivated and activated. In addition, these forces were determined using the Ansys finite element method that was detailed in Section 4.1. Figure 7 shows a comparison between the simulated and experimental total forces.

It can be seen that the magnetic force acting on the permanent magnet when it is in contact with one of the ends of the sliding chamber (locking status) was around $6.8 \mathrm{~N}$ whilst a magnetic force of about $2.5 \mathrm{~N}$ was required to start its actuation. These forces in association with the $5 \mathrm{~mm}$ offset that was allowed between the hinge of the side sliding link and the hinge of the top swivelling component enabled the new actuator to produce a torque output of about $34 \mathrm{mN}$.m.

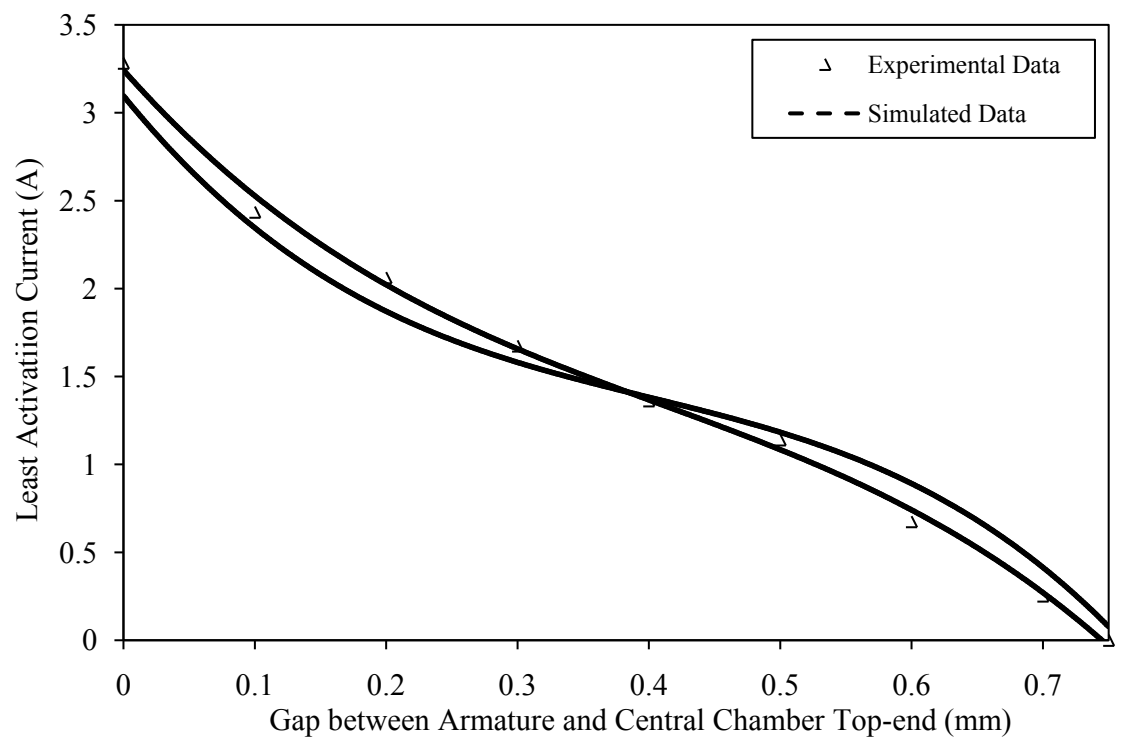

Figure 6. Least activation current versus gap between central chamber upper end and permanent magnet.

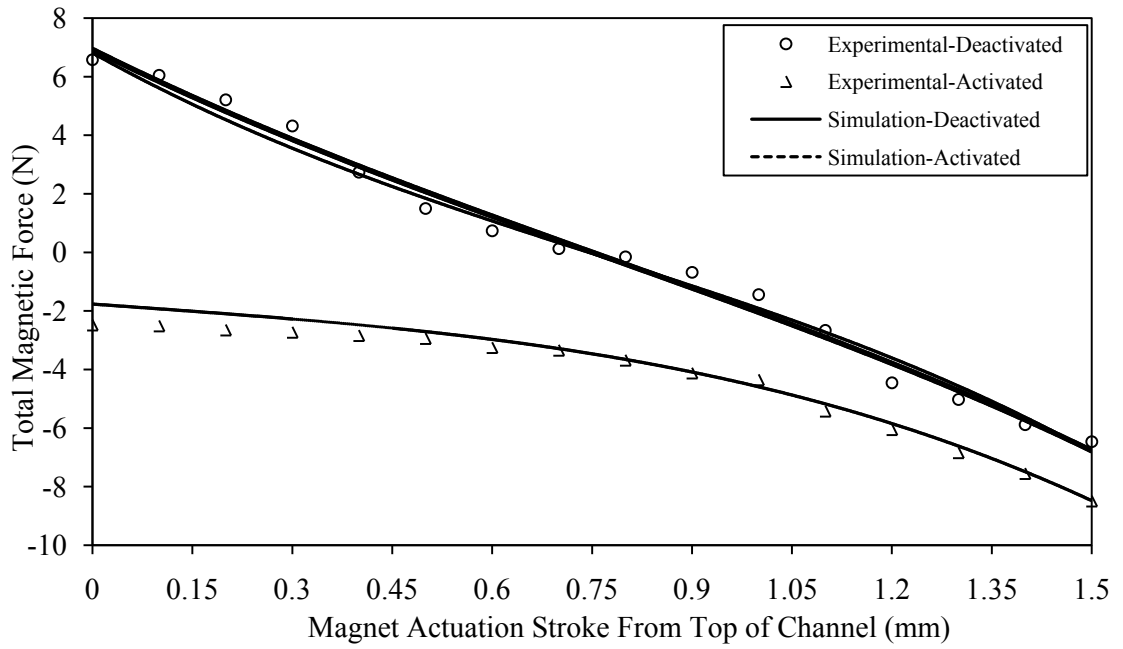

Figure 7. Total magnetic force on the permanent magnet along the actuation stroke. 


\subsection{Actuator Thermal Performance}

The thermal assessment of the new actuator was carried out, as detailed in Section 3.3, under a maximum current excitation of $6.7 \mathrm{~A}$ which is the current under which the optimum actuator performance was achieved. The results of this test are shown in Figure 8 where the temperature growth and decay are seen to follow certain paths over a period of about $200 \mathrm{~s}$. The $50^{\circ} \mathrm{C}$ maximum temperature target represents the maximum safe temperature that is allowed in SLS operations without causing damages to human tissues [22].

The duty cycle of the coil was then obtained using Equation (2) for which certain parameters were required including the coil surface area, maximum applied current and the temperature rise from the ambient level to the $50^{\circ} \mathrm{C}$ target together with the heat dissipation coefficient and the coil resistance. The first three parameters were substituted into the above equation as $S=358.032 \mathrm{~mm}^{2}, I_{\max }=$ 6.7 A and $\Delta T=23^{\circ} \mathrm{C}$, respectively. The heat dissipation coefficient, $\lambda$ was estimated using Equation (3) which was found to be 9.727 whilst the electrical resistance of the coil element, $R_{1}$ was measured immediately after the temperature began to drop from the $50^{\circ} \mathrm{C}$ level using a multi-meter and it was found to be approximately $1.6 \mathrm{Ohm}$. Accordingly, the duty cycle was estimated as $1.115 \times$ $10^{-3}$. This means that a heat dissipation time, $t_{2}$ would be around $89.7 \mathrm{~s}$ with the assumption of $t_{1}$ as $0.1 \mathrm{~s}$. As a result, the surgeon could actuate each single actuator about 4 times in every 3 minutes without breaching the safe heat generation level, which is a normal practice in most SLS operations.

\subsection{Summary of Actuator Performance}

The estimated overall performance of the new actuator is summarised in Table 2 , which is benchmarked against the performance of other actuators already reported in the literature for SLS applications. According to this table, the torque and force output of the new actuator were found to be adequate to generate the bending required by SLS tools. This was achieved when the new miniature size actuator was energised by a low voltage feed in comparison with the relatively large actuators (reported in Table 2), which employed large power units and complex power transmission (see for example, the fluidic actuator requirements, including the need for fluid tank).

Important design parameters such as the length, total diameter and weight of the new latching-type electromagnetic actuator, which are $31.6 \mathrm{~mm}, 10 \mathrm{~mm}$ and 23 grams, respectively should permit its application to optimise the function of SLS tools. In addition, the $30^{\circ}$ bending angle which is produced by the new actuator should enhance SLS tools articulation and adaptation to various SLS operation conditions. This articulation ability should be increased further if 3 to 4 new actuators are embedded at joints along the shaft of SLS tools, which should permit at least 6 degrees-of-freedom and $90^{\circ}$ to $120^{\circ}$ angulations in total. Furthermore, the results of the thermal tests have shown that considering conventional SLS surgical protocols, the new actuator is safe enough for operations in 
SLS environments. Finally, a conventional SLS tool was modified to enable its articulation and facilitate multi-degrees-of-freedom motions when three prototypes of the new electromagnetic actuator, which could be individually controlled, were embedded in tandem (back to front) along its shaft, as shown in Figure 9. In future work, the reliability and practical performance of the developed articulated SLS tool with its associated electromagnetic actuators would be the subject of an intensive programme of clinical trials.

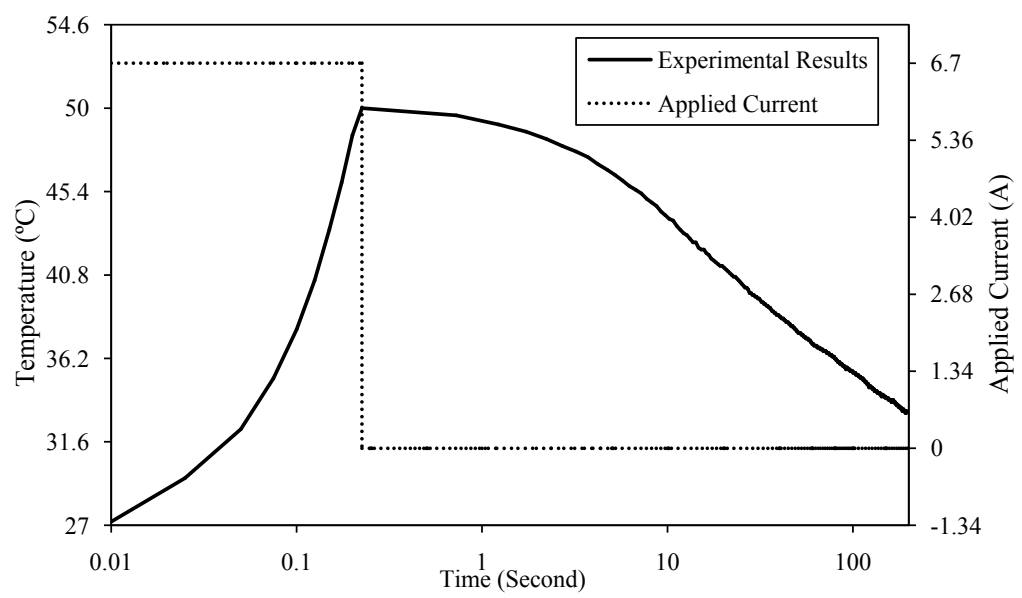

Figure 8. Temperature variation versus time for the new actuator under 6.7 A excitation.
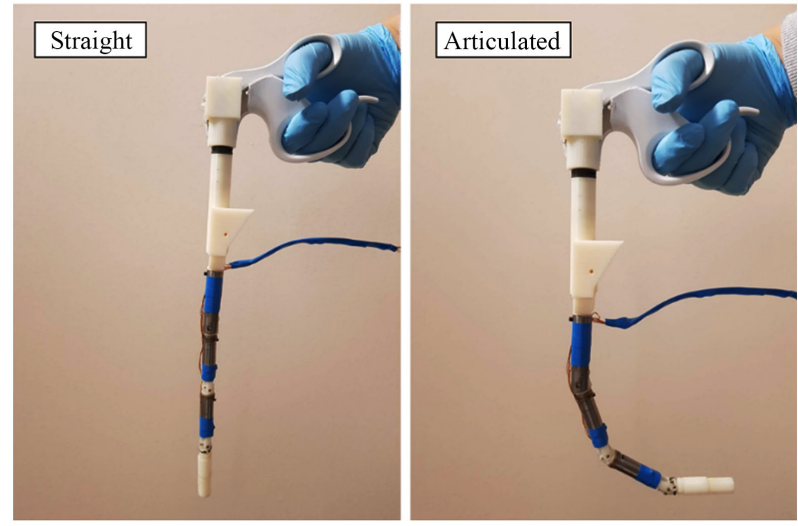

Figure 9. An SLS instrument prototype incorporating three new latching-type electromagnetic actuators (shown in its straight and articulated position).

Table 2. Performance of different actuators proposed for SLS applications.

\begin{tabular}{ccccc}
\hline Actuator Type & $\begin{array}{c}\text { Total } \\
\text { Length (mm) }\end{array}$ & $\begin{array}{c}\text { Outer } \\
\text { Diameter (mm) }\end{array}$ & $\begin{array}{c}\text { Torque } \\
\text { Output (mN.m) }\end{array}$ & $\begin{array}{c}\text { Bending } \\
\text { Angle }\end{array}$ \\
\hline Tendon Drive Actuator [23] & 35 & 12.5 & $5.7-17.5$ & $45^{\circ}$ \\
Double-Screw Drive Actuator [24] & 50 & 30 & High & $45^{\circ}$ \\
Pneumatic Drive Actuator [25] & 50 & 35 & N/A & $120^{\circ}$ \\
Fluidic Drive Actuator [26] & 78.4 & 9.6 & 26.39 & $125^{\circ}$ \\
$\begin{array}{c}\text { New Latching-Type } \\
\text { Electromagnetic Actuator }\end{array}$ & 31.6 & 10 & 34 & $30^{\circ}$ \\
\hline
\end{tabular}




\section{Conclusions}

This paper reports on a new latching-type electromagnetic actuator that is developed for applications in single-port laparoscopic surgery (SLS). Various experiments were conducted with the aim to evaluate the performance of the new electromagnetic actuator. The actuator performance was benchmarked against that obtained using finite element modelling techniques which were executed using Ansys Workbench software and the agreement between the two sets of results was found to be good. In particular, the employed finite element model was capable of predicting the actuator's least activation current, total magnetic force and thermal behaviour under various input conditions. This indicated that the developed finite element techniques would be suitable to design other electromagnetic-type actuators that could be developed for various engineering applications.

The performance of the developed new actuator was found to satisfy current requirements in SLS operation. In particular, the size and weight together with the torque and bending outputs of the new actuator were found to exceed those offered by several other actuators that were also aimed for SLS applications.

\section{Acknowledgements}

This work was supported by the Wellcome Trust through a translational medical research fund 120804894.

\section{Ethical Approval}

Not required.

\section{Conflicts of Interest}

The authors declare no conflicts of interest regarding the publication of this paper.

\section{References}

[1] Bulut, O. (2016) Annals of Laparoscopic and Endoscopic Surgery, 1, 38. https://doi.org/10.21037/ales.2016.11.20

[2] Fader, A. and Escobar, P. (2009) Gynecologic Oncology, 114, 157-161. https://doi.org/10.1016/j.ygyno.2009.05.020

[3] Stolzenburg, J., Kallidonis, P., Hellawell, G., Do, M., Haefner, T., Dietel, A. and Liatsikos, E. (2009) European Urology, 56, 644-650. https://doi.org/10.1016/j.eururo.2009.06.022

[4] Choi, B., Lee, K. and Lee, S. (2014) Journal of Laparoendoscopic \& Advanced Surgical Techniques, 24, 333-338. https://doi.org/10.1089/lap.2013.0497

[5] Ramirez, D., Maurice, M. and Kaouk, J. (2016) Urology, 95, 5-10. https://doi.org/10.1016/j.urology.2016.05.013

[6] Park, S., Bergs, R., Eberhart, R., Baker, L., Fernandez, R. and Cadeddu, J. (2007) Annals of Surgery, 245, 379-384. https://doi.org/10.1097/01.sla.0000232518.01447.c7

[7] Stolzenburg, J., Kallidonis, P., Oh, M., Nabi, G., Do, M., Haefner, T., Dietel, A., Till, 
H., Sakellaropoulos, G. and Liatsikos, E. (2010) Journal of Endourology, 24, 239-245. https://doi.org/10.1089/end.2009.0296

[8] Akshay, K. and Sameer, R. (2016) IOSR Journal of Dental and Medical Sciences, 15, 112-115. https://doi.org/10.9790/0853-15071112115

[9] Goel, A. (2012) Surgical Science, 3, 503-505. https://doi.org/10.4236/ss.2012.310100

[10] Le, H., Do, T. and Phee, S. (2016) Sensors and Actuators A: Physical, 247, 323-354. https://doi.org/10.1016/j.sna.2016.06.010

[11] Vitiello, V., Lee, S., Cundy, T. and Yang, G. (2013) IEEE Reviews in Biomedical Engineering, 6, 111-126. https://doi.org/10.1109/RBME.2012.2236311

[12] De Cristofaro, S., Stefanini, C., Pak, N., Susilo, E., Carrozza, M. and Dario, P. (2010) Sensors and Actuators A: Physical, 161, 234-244. https://doi.org/10.1016/j.sna.2010.04.028

[13] Lo, H. and Xie, S. (2012) Medical Engineering \& Physics, 34, 261-268. https://doi.org/10.1016/j.medengphy.2011.10.004

[14] Sliker, L., Ciuti, G. and Rentschler, M. (2015) Expert Review of Medical Devices, 12, 737-752. https://doi.org/10.1586/17434440.2015.1080120

[15] Jeong, S., Choi, H., Go, G., Lee, C., Lim, K., Sim, D., Jeong, M., Ko, S., Park, J. and Park, S. (2016) Medical Engineering \& Physics, 38, 403-410. https://doi.org/10.1016/j.medengphy.2016.01.001

[16] Ravaud, R., Lemarquand, G., Babic, S., Lemarquand, V. and Akyel, C. (2010) IEEE Transactions on Magnetics, 46, 3585-3590. https://doi.org/10.1109/TMAG.2010.2049026

[17] Kumar, A., Attaluri, A., Mallipudi, R., Cornejo, C., Bordelon, D., Armour, M., Morua, K., Deweese, T.L. and Ivkov, R. (2013) International Journal of Hyperthermia, 29, 106-120. https://doi.org/10.3109/02656736.2013.764023

[18] Kim, J. and Chang, J. (2007) IEEE Transactions on Magnetics, 43, 1849-1852. https://doi.org/10.1109/TMAG.2006.892289

[19] Lee, H., Ahn, J. and Kim, H. (2016) DApplied Sciences, 6, 288. https://doi.org/10.3390/app6100288

[20] Tennant Metallurgical Group Ltd., Chesterfield, United Kingdom (2014) Electromagnetic Test on Cast 80626. http://www.tenmet.co.uk

[21] Amazing Magnets, LLC, 5437 E La Palma Ave, Anaheim (2015) Magnet Grade Chart. https://www.amazingmagnets.com/magnetgrades.aspx

[22] Yarmolenko, P., Moon, E., Landon, C., Manzoor, A., Hochman, D., Viglianti, B. and Dewhirst, M. (2011) International Journal of Hyperthermia, 27, 320-343. https://doi.org/10.3109/02656736.2010.534527

[23] Newton, R.C., Noonan, D.P., Vitiello, V., Clark, J., Payne, C.J., Shang, J., Sodergren, M., Darzi, A. and Yang, G.Z. (2012) Surgical Endoscopy, 26, 2532-2540. https://doi.org/10.1007/s00464-012-2228-1

[24] Kobayashi, Y., Tomono, Y., Sekiguchi, Y., Watanabe, H., Toyoda, K., Konishi, K., Tomikawa, M., Ieiri, S., Tanoue, K., Hashizume, M. and Fujie, M. (2010) The International Journal of Medical Robotics and Computer Assisted Surgery, 6, 454-464. https://doi.org/10.1002/rcs.355

[25] Cianchetti, M., Ranzani, T., Gerboni, G., Nanayakkara, T., Althoefer, K., Dasgupta, P. and Menciassi, A. (2014) Soft Robot, 1, 122-131.

https://doi.org/10.1089/soro.2014.0001

[26] Chang, B., Chew, A., Naghshineh, N. and Menon, C. (2012) Smart Materials and Structures, 21, Article ID: 045008. https://doi.org/10.1088/0964-1726/21/4/045008 